\title{
ACUTE CEREBELLAR ATAXIA
}

\author{
BY \\ D. G. COTTOM \\ From The Hospital for Sick Children, Great Ormond Street, London
}

(RECEIVED FOR PUBLICATION NOVEMBER 29, 1956)

Acute cerebellar ataxia occurring in childhood is a definite clear-cut syndrome, yet only three cases appear to have been reported in the British literature (Batten, 1907; Taylor, 1913), although more recently cases with similar features have been included in wider surveys on encephalitis by Brewis (1954) and on ataxia by Shanks (1950). Typically the clinical picture is characterized by the sudden onset of ataxia in a previously well child. The infant staggers, falls and acquires a drunken, uncertain gait; there is often a gross intention tremor, staccato speech and frequently nystagmus. There are no signs of meningeal irritation, fever is absent and the infant remains alert with no alteration of consciousness. Despite these alarming signs the prognosis is generally favourable and recovery takes place over a period of time ranging from a few days to six months.

The object of this paper is to report seven further cases of acute cerebellar ataxia, to discuss those already reported and to draw attention to a syndrome which, if unrecognized, may result in disorders of a more serious nature being diagnosed.

\section{Case Reports}

M.B. 239854. A boy of 5 years, who had been progressing normally both physically and mentally, developed an urticarial rash and a low-grade fever. His mother thought he was incubating measles and kept him in bed for three days. He remained rather listless and after a week his temperature rose to $102^{\circ} \mathrm{F}$., but this resolved spontaneously without treatment. A week later he became unsteady on his legs and his mother noticed that he seemed unable to keep his eyes still. He was admitted to the Royal Hampshire County Hospital where he was found to be afebrile, but he was lethargic and had ataxia of the trunk and of all four limbs. There was nystagmus and a convergent strabismus, though the ocular fundi were normal; there was generalized hypotonia, and the reflexes were sluggish and the plantar responses were flexor. He had slight neck stiffness and a lumbar puncture showed a clear colourless fluid under normal pressure with 3 leucocytes and a protein content of $80 \mathrm{mg} . \%$.

He was transferred to The Hospital for Sick Children,
Great Ormond Street, with the provisional diagnosis of a cerebellar tumour. By this time the nystagmus had disappeared, but he remained apathetic and would only sit up if he were able to hold on to some support. The lower limbs remained ataxic, the deep reflexes were brisk and there was ill-sustained ankle clonus, together with extensor plantar responses.

Investigations included: Haemoglobin $89 \%$, W.B.C. 7,700 per c.mm., with a normal differential, B.S.R. $5 \mathrm{~mm}$. in one hour. A Mantoux test $(1: 1,000)$ was negative. Radiographs of the chest and skull were both normal. The C.S.F. on this occasion contained 10 W.B.C. per c.mm., $30 \mathrm{mg}$. protein per $100 \mathrm{ml}$. and $67 \mathrm{mg}$. sugar per $100 \mathrm{ml}$. A ventriculogram was entirely normal.

Over the next fortnight he continued to improve, the ataxia became less marked and normal muscle tone returned. When examined two months after the onset of the illness he was very well and the only residual sign was a slight intention tremor.

M.H. 254707. A boy was born on December 21, 1953. He was seen at the age of 22 months because of vomiting and a staggering, unsteady gait. He was the second child of healthy unrelated parents; he was born after a normal pregnancy and weighed $7 \mathrm{lb} .3 \mathrm{oz}$. at birth. $\mathrm{He}$ had begun to walk normally when 12 months old and had been steady on his feet until three weeks before admission when he developed a considerable tremor of both arms and legs. When this had been present for 10 days he began to vomit after most meals and lost weight.

On examination he was seen to be a small, wide-eyed little boy weighing only $19 \mathrm{lb}$. He was afebrile and there was no alteration of consciousness. The skin was rough and he was moderately dehydrated. There was a variable coarse nystagmus, the ocular fundi were paler than normal, but there was no papilloedema. A pronounced intention tremor was present in both arms, there was a tremulous fluttering of the eyelids and a definite rapid tremor involving the head and trunk. He was ataxic and unable to walk, although the power and sensation in his lower limbs appeared normal. There was considerable hypotonia of the trunk and legs, the knee and ankle jerks could not be obtained, but the plantar responses were extensor.

Investigations included radiographs of the skull and chest, both of which were normal. The haemoglobin was $88 \%(13.02$ g. per $100 \mathrm{ml}$.) and W.B.C. 8,400 per 
c.mm. with a normal differential. Lumbar puncture produced a clear fluid under normal pressure which contained no cells; the protein was $30 \mathrm{mg}$. per $100 \mathrm{ml}$. but one week later this had risen to $240 \mathrm{mg}$. per $100 \mathrm{ml}$. still without any cells. The sugar content was $78 \mathrm{mg}$. per $100 \mathrm{ml}$. on both occasions and the Wassermann reaction was negative. The B.S.R. was $2 \mathrm{~mm}$. in one hour and the electrophoretic strip showed a persistently low $\gamma$ globulin.

He gradually improved over the course of the next month, the nystagmus disappeared, the tremor became less marked and feeding became easier. After a further three weeks he began to walk again, to talk and to feed normally. When last seen, three months from the beginning of symptoms, he was vastly better and could walk unaided.

A.B. 248538. A.B., aged 18 months, was admitted to hospital because of a generalized tremor which had come on with alarming suddenness the day before. He was the first child of Jewish parents and previously had been entirely healthy. His milestones were normal and he had been walking since 1 year. There was no recent history of immunization or of contact with any infectious illness. The day before admission he was more fretful than usual and he held on to the furniture to. steady himself when walking. By the afternoon it was noticed that his arm shook when he attempted to suck his finger, and by the evening he was quite unable to stand. There was no vomiting and no alteration of consciousness.

When examined 24 hours after the onset of the illness he had a coarse intention tremor involving the entire body. He was ataxic and had a tremulous cry. There was universal hypotonia of limbs and trunk, although the reflexes remained brisk and symmetrical, and the plantar responses, extensor. Apart from a coarse nystagmus, the cranial nerves were normal. The ocular fundi and the ears showed no abnormality. Although there was some voluntary resistance to neck movement, no true meningism could be demonstrated. The temperature was $98 \cdot 6$ degrees $\mathrm{F}$.

Laboratory investigations included lumbar puncture which gave clear fluid under normal pressure, containing 43 cells per c.mm., mainly mononuclears, $30 \mathrm{mg}$. protein, $56 \mathrm{mg}$. \% sugar and $720 \mathrm{mg}$. \% chlorides. I_eucocytes numbered 7,400 (polymorphs $54 \%$, lymphocytes $46 \%$ ). A skull radiograph was normal. The Wassermann reaction was negative. The B.S.R. was $5 \mathrm{~mm}$. in one hour. A Mantoux test $(1$ in 1,000$)$ was negative. The electrophoretic pattern was normal. Complement-fixation tests for toxoplasmosis were negative. An E.E.G. (Dr. Cobb) gave a normal sleep record.

His condition worsened for 48 hours, the tremor became even wilder and he was only happy when lying flat. The reflexes were increasingly brisk and ankle clonus was obtainable for two/three days. He remained extremely ataxic for six weeks and was very difficult to feed, as any effort on his part provoked such gross tremor. Gradually he began to improve, the cells in the C.S.F. fell to 10 per c.mm. with a protein of $80 \mathrm{mg} . \%$.
Three months after the beginning of the illness he developed a squint with a right external rectus weakness and a lumbar encephalogram was carried out to exclude a posterior fossa tumour. It was entirely normal. He has continued to improve slowly, but six months after the onset he is still unable to walk and it appears likely that there is considerable mental impairment.

J.E. 178295. J.E., a girl of 5 years, was admitted on January 7, 1952, because of a sudden onset of tremor and inability to walk. In the past she had received treatment for recurrent attacks of pyelitis and her appendix had been removed. Since that time she had been well until a week before coming to hospital, when she developed an upper respiratory infection with coryza, cough and fever. This was settling, although two days before the onset of neurological symptoms her temperature had been up to $103^{\circ} \mathrm{F}$. On the day of admission she became very tremulous and was unable to stand, as any movement provoked a gross intention tremor. She was ataxic and her speech was slurred, she had vomited, but was free from headaches except when she coughed.

Examination revealed a fair-haired girl with a rather immobile facies. She was afebrile, alert and cooperative but frightened to move. There was no neck stiffness and the ocular fundi were normal; there was nystagmus on looking either to the extreme right or left. All limbs were hypotonic yet the reflexes were brisk, the knee jerks pendular and the plantar responses flexor. There was no muscle tenderness, but gross incoordination and intention tremor of the legs and arms. Sensation was normal.

Lumbar puncture produced a clear C.S.F. with 27 cells, mainly lymphocytes, a protein of $30 \mathrm{mg}$. and sugar $76 \mathrm{mg}$. \%. A blood count gave: Haemoglobin $95 \%$, W.B.C. $8,600(53 \%$ polymorphs, $41 \%$ lymphocytes, $6 \%$ monocytes).

She became worse for the first 48 hours in hospital, the tremor became more exaggerated, the vomiting continued and an intragastric drip was necessary. In addition she was unable to pass urine and had to be catheterized. By the third day she was beginning to improve, and although she complained that the bed was rotating she was no longer sick. A further lumbar puncture contained 6 cells, with $20 \mathrm{mg}$. protein and normal sugar and chlorides.

Gradually over a month her speech returned to normal, the ataxia and tremor diminished, although the hypotonia and lack of coordination in the performance of fine movements persisted. Not for a further two months had her gait returned to normal and it was 15 weeks from the onset before she was discharged from convalescence.

J.B. 252728. A boy, born on September 9, 1951, was seen at the age of 4 because nine days previously he had fallen on getting out of bed and had been unsteady on his feet ever since. On the first day of the illness he had had numerous falls and had vomited a number of times. His limbs appeared weak, particularly the left arm, he continued to stagger and remained very miser- 
able. His temperature was normal, he had no headache and there was no disturbance of sphincter control.

He was a well-nourished 4-year-old who held his head over to the right, and walked with an unsteady gait. There was hypotonia of all limbs, but the tremor and inability to perform fine movements was most marked in the left arm. There was no meningism, no nystagmus and the ocular fundi were normal. All the tendon refiexes were brisk and the plantar responses were extensor. Investigations were essentially normal. The B.S.R. was $4 \mathrm{~mm}$. in one hour, radiographs of the skull and chest showed no abnormality, the Wassermann reaction was negative, the C.S.F. contained 3 cells per c.mm. with normal protein and sugar content.

During the next week he began to improve, he became much happier, he staggered less and there was no more vomiting. Six weeks after the onset of the illness his gait had returned to normal, the tendon reflexes were no longer brisk and the plantar responses were flexor. He has been seen at intervals over the last two years and he remains entirely well.

J.R. 251293. A girl of $10 \frac{1}{2}$ years was admitted to The Hospital for Sick Children, Great Ormond Street, because for the past week she had been unsteady on her feet and had been vomiting. Two months previously she had developed a squint and had suffered from double vision, but this cleared after one week and she had been well until the sudden onset of ataxia and vomiting. She was the third child of healthy parents. At the age of 4 she had had staphylococcal pneumonia, but had otherwise been well. On examination she was found to be rather drowsy, the pupils reacted sluggishly to light, there was a bilateral external rectus weakness and a poorly sustained nystagmus. The ocular fundi were normal. There was slight facial asymmetry, the mouth drooping on the left. The tone was normal, but there was considerable ataxia with dysdiadokokinesia of all four limbs. The reflexes were symmetrical the knee jerks were pendular and the plantar responses flexor.

She continued to be rather drowsy and vomited if she attempted to sit upright. At no time did she complain of headache, there was no fever and no signs of meningism. Laboratory investigations showed: Haemoglobin $88 \%(13.02 \mathrm{~g}$. per $100 \mathrm{ml}$.), W.B.C. 8,300 (neutrophils $78 \%$, lymphocytes $22 \%$ ). The C.S.F. was clear and colourless, it contained 10 white cells per c.mm., $120 \mathrm{mg}$. per $100 \mathrm{ml}$. of protein and $69 \mathrm{mg}$. per $100 \mathrm{ml}$. sugar. An E.E.G. by Dr. Dickson at the National Hospital, Queen Square, showed marked asymmetry between the two sides suggesting that the left hemisphere was probably involved. In an effort to exclude a posterior fossa tumour a lumbar encephalogram and a ventriculogram were done and were both normal. After one month in hospital she had improved considerably, she was much more alert, there was no vomiting and she was able to walk unsupported on a wide base. Nystagmus was still present, there was a marked intention tremor but the ataxia was much less pronounced.

When seen four months after the onset of the illness she had returned to school and was able to walk a mile there and back each day. On examination a mild residual cerebellar deficit was apparent, but this appears to be improving and causes her no inconvenience.

C.H. P.P. C.H. was a doctor's daughter of 18 months who had been well until a week ago when she began to vomit. The next day she was noticed to be walking unsteadily and by the evening of that day there was a pronounced stagger. She continued to vomit that night and by morning was unable to stand. The temperature remained normal, there was no meningism, no nystagmus and the ocular fundi showed no abnormality. She held her head over to the left and had a left internal strabismus. The tendon reflexes were normal and the plantar responses extensor. After three days she began to improve, and after a further two days she was able to walk with a tottery, wide-based gait. In view of her rapid improvement she was not admitted to hospital and no investigations were carried out. Over the next two weeks she made a complete recovery and there are no residual sequelae.

\section{Reported Cases}

Batten writing in 1907 was the first to draw attention to this syndrome in Great Britain. He reported the case of a boy of 11 years who without preceding illnesses suddenly developed a headache and ataxia. He was found to have nystagmus and some loss of tone, together with active knee jerks; there was a rapid improvement so that in three weeks the child was completely recovered. Claude and Schaeffer (1911) published their findings in two cases of ataxia seen in Paris. The second of these, a child of $3 \frac{1}{2}$ years, showed the typical picture of acute cerebellar ataxia with recovery after three months. Taylor (1913) described the syndrome in two patients aged 7 and 10 years and he contrasted their sudden onset and benign prognosis with the poorer outlook in two other patients with 'progressive congenital cerebellar ataxia'. The same year Merle (1913) reported the case of a boy of 6 years who recovered after an illness lasting three weeks characterized by a sudden onset, ataxia and tremor, and in which the cerebrospinal fluid contained an excess of protein.

Griffiths in three papers $(1916,1920,1921)$ described four additional cases and reviewed the literature up to that time. Of the 31 cases he quotes, all but six had had an acute infectious illness just before the onset of neurological symptoms. They differ from acute cerebellar ataxia in a number of features and fall more readily into the group of post-infective encephalitides. With the exception of six cases (two of Griffiths' own and those of Taylor, 1913; Batten, 1907; and Seham, 1919) there had been a preceding illness such as measles, pertussis, typhoid or varicella which had become worse with 
the onset of convulsions or loss of consciousness. This was followed by varying degrees of ataxia, but in addition there were other signs of widespread involvement of the brain, paresis, loss of sphincter control, aphasia and disturbances of sensation were often present. The condition persisted for many months and frequently left residual mental changes. Although in acute cerebellar ataxia there may be evidence that the cerebrum is partially involved, the other signs of brain damage are not seen and this, together with the more favourable prognosis, is sufficient reason for regarding it as a separate entity.

Barbonneix (1928) reported five cases of ataxia occurring in childhood, of which three appear to be typical cases of acute cerebellar ataxia.

In 1944 Klingman and Hodges reported seven cases of acute ataxia of unknown origin occurring during the preceding three years in New York. Six of these appear to be typical examples of acute cerebellar ataxia and one is of particular interest in that although there was complete recovery of function the illness was followed by a conduct disorder of moderate severity. One case (5. F.M.) is unusual in that he had convulsions associated with ataxia, but no other signs of cerebellar dysfunction, and for that reason has not been included in this series.

The single case of Keller and Karelitz (1948) is interesting in that both clinical and E.E.G. findings were suggestive of a diffuse process involving the cerebellum and mid-brain. Both this and the comparatively prolonged illness is very similar to Case A.B.

An excellent account is given by Thieffry, Martin and Arthuis (1953). They describe eight cases seen between 1948 and 1951 in Paris, all of which made a complete recovery. They comment particularly on the pleocytosis sometimes observed in the C.S.F. and on the favourable outcome. A recent paper of Berglund, Mossberg and Rydenstam (1955) from Stockholm reports the findings in five typical cases and is particularly important for the virus studies carried out on them.

It has been possible to extract from the literature a total of 33 cases whose pattern is similar and which appear to be examples of the same syndrome. These, together with seven of our own cases, form the basis for this analysis.

\section{Symptomatology}

Onset. The onset in all cases is acute. Often the disease comes on with such devastating suddenness that the parents are able to state the hour at which it began. It attacks previously healthy children usually of the toddler age group and within six to 12 hours they are completely incapacitated. In one case reported by Thieffry et al. the onset of ataxia came on with such rapidity during a railway journey from Baden-Baden to Paris that the family assumed that their $3 \frac{1}{2}$-year-old son must indeed be drunk. Shanks (1950), in his review of 39 cases of cerebellar ataxia occurring between 1929 and 1949 in Glasgow, pointed out that in general a sudden onset was associated with a good prognosis, whereas those that came on insidiously tend to do badly. Although six out of 17 cases with an acute onset had suffered from other illnesses in the preceding three weeks and must be considered as post-infective encephalitides, a number of his cases are doubtless examples of acute cerebellar ataxia, but unfortunately no case histories are given.

Ataxia. The initial complaint is usually that the infant can no longer walk normally. He either staggers and falls or else refuses to make any attempt to walk. In those who are only moderately affected there is an unsteady, drunken gait with the feet wide apart. In others the ataxia may be so gross that they are only comfortable when lying prone. Case A.B. became immediately distressed if even his head was raised from the pillow. In all cases there was a total inability to adapt to changing postures during voluntary movements. This was frequently the first noticeable symptom and it persisted when other symptoms had cleared.

Tremor. This varied from a mild intention tremor to gross rhythmic movements involving all parts of the body. In particular there was a considerable tremor of the upper limbs, in one case of sufficient magnitude to prevent the infant having his thumb in his mouth. There was a tremor of the head, especially on attempting ocular fixation and when being fed. Perhaps the most constant tremor is that involving the eyes; there was a pronounced tremulous fluttering of the eyelids in three of our cases. This has been noted in numerous other cases, and like the infant reported by Keller and Karelitz (1948), it may persist even during sleep. In addition there may be dissociated tremulous movements of the eyes which become gross when trying to fix on an object.

Nystagmus. This was present in five of our cases, but was only commented on in 13 out of 33 cases in the literature. In our cases any attempt at ocular fixation provoked a coarse oscillatory nystagmus of a cerebellar type. Nystagmus disappears early in the course of recovery and may no longer be obtainable even when a considerable degree of ataxia is still present. 
Hypotonia. Hypotonia may be very marked. It was very obvious in four of our seven cases and was a consistent finding in those reported by Thieffry et al. It was a notable feature in 12 cases taken from the literature and was present in a further three. In most instances this lack of muscle tone was associated with increased knee and ankle reflexes. These were usually symmetrical and occasionally ankle clonus could be elicited when the disease was at its height (in two of our cases and two in the literature, Goldwyn and Waldman, 1953).

Other Symptoms. Speech was affected in more than half the cases; in the older children a staccato tremulous enunciation developed, whilst in the younger age group they made little attempt to speak, but a tremor was often apparent in the cry. A squint was present in four of our cases and it received comment in eight of those taken from the literature. It was usually due to an external rectus weakness, it often appeared relatively late in the illness, and persisted for only a short time.

It is perhaps equally important to draw attention to the absence of certain symptoms and signs which serve to distinguish acute cerebellar ataxia from similar conditions. Even among the older children headache was an uncommon symptom. It was absent in all our cases and it is only recorded in four cases in the literature, but it is important to realize that over half the cases are below the age of 3 years and are perhaps unlikely to complain of headache. In none of the cases were there signs of meningism. A number of children were disinclined to move their heads because of the vertigo it caused, but none had any demonstrable neck rigidity. Likewise, Kernig's and Lasègue's signs were constantly negative in our cases and in those from the literature in which they were tested.

Again fever was remarkable by its absence. In Case J.E. the child had an upper respiratory infection and a temperature of $103^{\circ} \mathrm{F}$. two days before the onset of tremor and ataxia, although it was normal by the time she was admitted to hospital. Only two of 33 cases taken from the literature were febrile; Taylor's (1913) second case ran a fever between 99 and $102^{\circ} \mathrm{F}$., but it is perhaps significant that this child had an associated otitis media. Again the case (A.G.) reported by Klingman and Hodges (1944) had tonsillitis and a temperature of $102^{\circ} \mathrm{F}$. at the onset of neurological symptoms. It would seem probable that on the occasions when the child has been febrile there have been coincident illnesses to account for it and that it is not part of the symptomatology of acute cerebellar ataxia.

\section{Investigations}

Since this survey covers cases reported over a period of 50 years, their mode of investigation naturally varies very widely. In the earlier studies the possibility of syphilis or tubercle being causal agents were excluded by finding negative Wassermann and Mantoux reactions. In the more recently reported cases greater emphasis has been placed on virus studies and on the findings of the electroencephalogram.

Haematology. Data are available in only a few cases. There is no evidence of anaemia. The white blood count is slightly raised, average 10,000 cells per c.mm., but the differential counts showed a normal distribution. The blood sedimentation rate as recorded in seven cases was always within the normal range.

C.S.F. Reports of the composition of the cerebrospinal fluid are available from a total of 30 cases. Characteristically it was a clear, colourless fluid under normal pressure. The white cell content varied between 0 and 90 lymphocytes per c.mm., although in the majority of cases it was normal and only six cases had counts of over 10 cells per c.mm. The protein was usually within normal limits at the initial lumbar puncture, but when subsequent examinations of the C.S.F. were carried out one to four weeks after the onset the number of cells had frequently fallen and the protein content risen considerably. In Case M.H. no cells were present at any time; 10 days after the onset of symptoms the protein was $30 \mathrm{mg}$. per $100 \mathrm{ml}$., a week later it had risen to $240 \mathrm{mg}$. per $100 \mathrm{ml}$. and after one month it was $140 \mathrm{mg}$. per $100 \mathrm{ml}$. Again, J.R.'s initial protein level rose from $40 \mathrm{mg}$. per $100 \mathrm{ml}$. to $120 \mathrm{mg}$. per $100 \mathrm{ml}$. during the first fortnight of her illness. This change receives little comment in the literature and this is probably due to the disinclination to perform more than a single diagnostic lumbar puncture. It was found in three of our cases and is reminiscent of the pattern found in poliomyelitis. The sugar and chloride contents were always within the normal range whenever they were recorded.

Radiological Studies. Radiographs of the skull were examined in a total of 22 cases and no abnormality was found on any occasion. Ventriculography was carried out in three of our cases and in a further six reported in the literature. The majority were entirely normal. Keller and Karelitz's case showed 'slight cortical atrophy' and Klingman and Hodge's Case 6 was said to show a moderate degree of atrophy. 
Electro-encephalogram. This was studied in 14 cases and was normal in eight. Of the others, four showed a slow, 4-5 cycles per second rhythm, especially over the occipital region, and one of these had reverted to normal when re-examined two weeks after the beginning of the illness (Berglund et al.). Keller's case was interesting in that the E.E.G. showed a diffuse process with the maximum damage in the mesencephalic region, an observation which was in accord with the clinical findings.

Toxoplasmosis was considered as a possible aetiological agent, but both the dye and complement-fixation tests were negative in three cases in which they were carried out (Cases A.B. and M.M. and Case 5 reported by Berglund et al.). The most fruitful investigations have been in the field of virus studies and they are considered in the section on aetiology.

\section{Aetiology}

Despite the small number of cases which have been described this syndrome appears to be widespread both in time and space. For the last 50 years sporadic cases have been reported from time to time occurring both in America and throughout Europe. No common aetiological factor has come to light, but the appearance of cases of acute cerebellar ataxia does seem to have been more common during epidemics of influenza, encephalitis lethargica and poliomyelitis. It may occur in children of all ages. The youngest in this series was 15 months and the eldest 11 years. Half the cases were under the age of 3 at the time of onset, and only six out of 40 were over 5 . The condition affects primarily the pre-school child and as yet no case of infection by contact has been reported. Likewise previous illness does not appear to play any part in its aetiology nor, unlike the progressive forms of cerebellar ataxia (Friedreich's, Sanger Brown, etc.), can any hereditary linkage be discerned.

Acute cerebellar ataxia begins with a startling suddenness which is suggestive of an infection and this, together with its similarity to poliomyelitis and the post-infective encephalitides, has focused attention on the possibility of its being yet another virus disease. Klingman and Hodges (1944) tested the serum of four of their patients for neutralizing antibodies against the viruses of St. Louis encephalitis and eastern equine encephalomyelitis and in two others they looked for evidence of infection by louping ill, lymphocytic choriomeningitis, western equine encephalomyelitis and herpes simplex-all with negative results.

The work of Berglund et al. is interesting. In two of their cases (Nos. 3 and 4) Type I (Brunhilde) poliomyelitis was found. In Case 3 complement fixation was positive for all three types of poliomyelitis, but neutralizing antibodies were obtained against Type I only. Examination of the faeces showed a growth of cytopathogenic agent Type I. When examined six months after the illness the complement-fixation test had become negative. Similar results were obtained in Case 4, and in Cases 1, 2 and 5 the complement-fixation reaction was positive against influenza $\mathbf{A}$ and $\mathbf{B}$, although in these cases significant changes of titre could not be demonstrated so that their aetiological role remains unproven. It is of interest that whereas the cases from which poliomyelitis was isolated ran a mild benign course, those in which influenza was implicated ran a more protracted course.

Virus studies were undertaken in three of our cases; in two no virus was isolated, but in the third a cytopathogenic growth was observed but the virus has not as yet been identified.

\section{Diagnosis}

First it is essential to exclude the possibility of a posterior fossa tumour. Four of our seven cases were admitted with this as the most likely diagnosis. The history of a child with a neoplasm is generally one of insidious or intermittent progression, with the gradual addition of symptoms over the course of weeks. It must be very unusual for a tumour to announce its presence with the devastating suddenness typical of acute cerebellar ataxia. The suggestion that such an acute onset might be due to haemorrhage into the substance of a tumour is put forward more often by the diagnostically destitute than it is found by the pathologist. - Secondly, by the time a tumour causes the gross disturbance of cerebellar function found in acute cerebellar ataxia it is likely to cause some signs of raised intracranial pressure, which may be detected in the ocular fundi or in the radiograph of the skull. Nevertheless on occasion the possible presence of a tumour cannot be ruled out and it is necessary to resort to air studies. The advisability of performing this investigation in the presence of whatever agent is causing acute cerebellar ataxia has been questioned. Recognition of the existence of this syndrome obviated its necessity in three of our cases, but the others in which it was carried out did not seem to be adversely affected by ventriculography.

Poliomyelitis is difficult to differentiate, a fact which is perhaps not surprising as there is some evidence that the virus of poliomyelitis may also be responsible for acute cerebellar ataxia. Even if 
this is true, the symptomatology is different, the patient with poliomyelitis has been ill for two or three days before the onset of neurological symptoms, he is usually febrile, complains of headache and has signs of meningism. In a recent case of 'cerebellar' poliomyelitis confirmed by virus studies, the child had a temperature of $102^{\circ} \mathrm{F}$. associated with moderate neck rigidity and appeared much more ill than those with acute cerebellar ataxia, although his tremor and unsteadiness were mild in comparison.

Labyrinthine ataxia is more easily distinguished. It is more a loss of static equilibrium, there is rotatory nystagmus and the patient tends to fall to the side of the lesion, the symptoms being made worse by closing the eyes. There may be an associated deafness or discharge from the ear and tests of labyrinthine function will be abnormal.

Acute demyelinating disease of the Schilder type may come into the differential diagnosis. Here symptoms may come on rapidly, but over the course of days rather than hours as in acute cerebellar ataxia. Visual failure together with mental deterioration, convulsions and weakness of all limbs usually serve to distinguish it, the demyelination affecting primarily cerebral cortex rather than cerebellum. Likewise the cerebellar degenerations should cause little confusion, and their slow progressive nature in persons with a family history of nervous disease enables the diagnosis to be clarified.

The question of trauma or of poisoning will usually be excluded by the absence of a history, but it is well to ask direct questions concerning the possible ingestion of heavy metals, insecticides similar to D.D.T. and perhaps of alcohol.

Recently Burgstedt (1955) has reported two cases of ataxia with loss of muscle tone following an overdose of piperazine during a course of treatment for threadworms. In general the diagnosis of acute ataxia is not difficult once its existence as a syndrome is recognized.

\section{Prognosis}

The outlook with regard to life was good in all cases. The average duration of the illness was two months, some cases were so slightly affected that recovery was complete within one week, whilst in others signs could be detected as long as eight months after the onset. Unlike the post-infective encephalitides sequelae are comparatively rare. In one of our cases (A.B.) there appears to be considerable mental impairment, and eight months after the beginning of his illness there is marked lack of response to both visual and auditory stimuli. This is unusual and it is perhaps significant that the acute phase was extremely severe and was associated with a high pleocytosis in the cerebrospinal fluid.

Residual signs of incoordination could be detected up to one year after the onset in four cases, but these were usually mild and did not interfere with normal activities in any way. Behaviour problems were a cause of concern in five cases and in one (Klingman and Hodges, G. S.) it was of sufficient severity to require institutional treatment. The other four children showed mild conduct disorders and in particular emotional reactions to momentary separation from their parents. In this last group it is difficult to decide whether the alteration in temperament is due primarily to the illness or to a period in hospital at an age when children are known to react badly to parental separation. Overall in 40 cases, one appears to have suffered serious brain damage and one has developed a moderately severe behaviour disorder. Full recovery has been the rule in the other 38 children, although some have continued to improve for as long as one year after the onset.

\section{Discussion}

The term 'acute ataxia' was first used by Leyden in 1891 and cases similar to those reported here have been described as examples of the 'acute ataxia of Leyden'. In fact the two cases described by Leyden were similar only with regard to the sudden onset of ataxia, they had in addition pareses, with disorders of sensation and showed no tendency to recover after periods up to two years. Acute cerebellar ataxia is probably the most convenient name to denote this syndrome since it is purely descriptive and does not imply any particular aetiology. On the other hand, it may be criticized in that it is equally applicable to cases of cerebellar ataxia coming on suddenly in degenerative diseases of the central nervous system and perhaps the best term is that used by the French workers. They call it 'l'ataxie cerebelleuse aigue curable de l'enfant'.

Acute cerebellar ataxia is fortunately not a fatal condition so that its pathology is unknown. By analogy with cases of encephalitis following measles, rubella, etc., it is possible that there is perivenous demyelination affecting primarily the cerebellum. Recently in a survey involving over 1,000 cases of neurological illness following infectious disease, Miller (1956) and his co-workers were struck by the similarity of the changes in the nervous system produced by a wide variety of different infections. Not only were the clinical pictures following measles, varicella and rubella strikingly similar, but from the histopathological point of view they were indistinguishable. Moreover, identical illnesses may 
follow non-specific infections, smallpox vaccination or serum sickness. Miller points out that it is probable that there is some common factor in pathogenesis between the specific initiating infection and the non-specific end-result in the nervous system and that such a common factor may be anaphylactic sensitization.

This concept of neuro-allergy receives support from the experimental work of Kabat, Wolf and Bezer (1949), Lumsden (1956) and others. It is known that parenteral injections of sterile brain tissue in a viscous paraffin oil base with heat-killed tubercle bacilli as adjuvants are capable of producing changes in the brain similar to those found in encephalitis. Adjuvants alone or similar systems with tissues other than brain are incapable of provoking this reaction. Lumsden in a recent review concludes that this work has shown that introduction of a definite biochemical system is capable of producing severe disease in the brain which is of an inflammatory type.

It is perhaps a far cry to suggest that acute cerebellar ataxia is due to a variety of agents, possibly viruses, which in the appropriate hypersensitive host are capable of causing an inflammatory lesion centred primarily in the cerebellum. There are, however, a number of facts which tend to favour this hypothesis. First, no single virus has been implicated as the cause of acute cerebellar ataxia, though it occurs more commonly when epidemics of poliomyelitis, influenza, or encephalitis lethargica are prevalent. The Scandinavian workers produced good evidence to suggest that both the poliomyelitis and influenza viruses were involved. In three cases there was a distinct rise in the protein content of the C.S.F. during the course of the illness with little change in the number of cells; in one case it was $240 \mathrm{mg}$. per $100 \mathrm{ml}$. This is perhaps suggestive of an exudative phase with capillary damage in the absence of inflammation. Together with the extremely sudden onset characteristic of 'allergic disorders' these facts lend some support to the hypersensitivity theory.

So far no specific treatment appears to have helped these patients. In two of our cases various antibiotics were given to prevent intercurrent infection, but were without effect on the neurological symptoms. Case A.B. had a course of Vitamin $B_{12}$ in high dosage, but this produced no change. The use of cortisone or A.C.T.H. would seem to be worthy of trial in these cases.

\section{Summary}

The condition of acute cerebellar ataxia is described. It is characterized by the dramatic suddenness of its onset in a previously well child. There is ataxia, tremor and frequently nystagmus, muscle tone is diminished although the reflexes are usually brisk. The child is afebrile and is free from headache or meningism. Sensation is unaffected.

Seven cases are reported and these, together with 33 taken from the literature, are analysed. It occurs principally in children under 5 years and recovery is usually complete in two or three months. Rarely the syndrome may persist for considerably longer and in two cases there is evidence of permanent brain damage.

The chief cause of diagnostic confusion is a posterior fossa tumour. The rapid onset of symptoms and the lack of signs of raised intracranial pressure favour the diagnosis of acute cerebellar ataxia.

There is some evidence that this syndrome is due to an abnormal reaction of the central nervous system to the viruses of poliomyelitis and influenza. The possible role of cortisone in treatment is discussed.

I am very grateful to the physicians of The Hospital for Sick Children, Great Ormond Street, for permission to report cases which were under their care. In particular I am indebted to Professor A. A. Moncrieff and to Dr. Paul Sandifer for their help and encouragement.

\section{REFERENCES}

Babonneix, L. (1928). Rif. med., 44, 694

Batten, F. E. (1907). Trans. clin. Soc., Lond., 40, 276.

Berglund, G., Mossberg, H. O. and Rydenstam, B. (1955). Acta paediat. (Uppsala), 44, 254.

Brewis E. G. (1954) Brit. med J, 1, 1298

Burgstedt, H. J. (1955). Münch. med. Wschr., 97, 531.

Claude, H. and Schaeffer, H. (1911). Bull. Soc. méd. Hôp. Paris, 3 ser., 31, 192.

Goldwyn, A. and Waldman, A. M. (1953). J. Pediat., 42, 75.

Griffith, J. P. C. (1916). Amer. J. med. Sci., 151, 24.

- (1920). Amer. J. Dis. Child., 20, 82.

(1920). Amer. J. Dis. Child., 20, 82.

Kabat, E. A., Wolf, A. and Bezer, A. E. (1949). J. exp. Med., 89, 395.

Keller, M. J. and Karelitz, S. (1948). Pediatrics, 1, 754.

Klingman, W. O. and Hodges, R. G. (1944). J. Pediat., 24, 536

Leyden, E. (1891). Z. klin. Med., 18, 576.

Lumsden, C. E. (1956). Proc. roy. Soc. Med., 49, 148.

Merle, P. (1913). Bull. Soc. méd. Hôp., Paris, 3 ser., 36, 325.

Miller, H. (1956). Proc. roy. Soc. Med., 49, 139.

Miller, H. (1956). Proc. roy. Soc. Med., 49,

Shanks, R. A. (1950). Archives of Disease in Childhood, 25, 389.

Taylor, E. S. (1913). Guy's Hosp. Rep., 67, 98.

Thieffry, S., Martin, C. and Arthuis, M. (1953). Arch. franc. Pédiat., $10,14$. 